\title{
Article
}

\section{Aerobic Continuous and Interval Training under Hypoxia Enhances Endurance Exercise Performance with Hemodynamic and Autonomic Nervous System Function in Amateur Male Swimmers}

\author{
Sung-Woo Kim ${ }^{1}{ }^{\mathbb{D}}$, Won-Sang Jung ${ }^{1}{ }^{\mathbb{D}}$, Jeong-Weon $\mathrm{Kim}^{2}{ }^{2}$, Sang-Seok Nam ${ }^{3}$ and Hun-Young Park ${ }^{1,4, * \mathbb{D}}$ \\ 1 Physical Activity and Performance Institute, Konkuk University, 120 Neungdong-ro, Gwangjin-gu, \\ Seoul 05029, Korea; kswrha@konkuk.ac.kr (S.-W.K.); jws1197@konkuk.ac.kr (W.-S.J.) \\ 2 Graduate School of Professional Therapy, Gachon University, 1332 Seongnam-daero, Sujeong-gu, \\ Seongnam-si 13306, Korea; zeezone@gachon.ac.kr \\ 3 Taekwondo Research Institute of Kukkiwon, 32 Teheran7gil, Gangnam-gu, Seoul 06130, Korea; \\ play_data@naver.com \\ 4 Department of Sports Medicine and Science, Graduate School, Konkuk University, 120 Neungdong-ro, \\ Gwangin-gu, Seoul 05029, Korea \\ * Correspondence: parkhy1980@konkuk.ac.kr; Tel.: +82-2-2049-6035
}

check for updates

Citation: Kim, S.-W.; Jung, W.-S.; Kim, J.-W.; Nam, S.-S.; Park, H.-Y. Aerobic Continuous and Interval Training under Hypoxia Enhances Endurance Exercise Performance with Hemodynamic and Autonomic Nervous System Function in Amateur Male Swimmers. Int. J. Environ. Res. Public Health 2021, 18, 3944. https:// doi.org/10.3390/ijerph18083944

Academic Editors: Matteo Cortesi, Sandro Bartolomei, Giorgio Gatta and Tomohiro Gonjo

Received: 25 February 2021

Accepted: 7 April 2021

Published: 9 April 2021

Publisher's Note: MDPI stays neutral with regard to jurisdictional claims in published maps and institutional affiliations.

Copyright: (c) 2021 by the authors. Licensee MDPI, Basel, Switzerland. This article is an open access article distributed under the terms and conditions of the Creative Commons Attribution (CC BY) license (https:// creativecommons.org/licenses/by/ $4.0 /)$.

\begin{abstract}
Hypoxic training is often performed by competitive swimmers to enhance their performance in normoxia. However, the beneficial effects of aerobic continuous and interval training under hypoxia on hemodynamic function, autonomic nervous system (ANS) function, and endurance exercise performance remain controversial. Here we investigated whether six weeks of aerobic continuous and interval training under hypoxia can improve hematological parameters, hemodynamic function, ANS function, and endurance exercise performance versus normoxia in amateur male swimmers. Twenty amateur male swimmers were equally assigned to the hypoxic training group or normoxic training group and evaluated before and after six weeks of training. Aerobic continuous and interval training in the hypoxia showed a more significantly improved hemodynamic function (heart rate, -653.4 vs. -353.7 beats $/ 30 \mathrm{~min}$; oxygen uptake, -62.45 vs. $-16.22 \mathrm{~mL} / \mathrm{kg} / 30 \mathrm{~min}$; stroke volume index, 197.66 vs. $52.32 \mathrm{~mL} / 30 \mathrm{~min}$ ) during submaximal exercise, ANS function (root mean square of successive differences, 10.15 vs. 3.32 ms; total power, 0.72 vs. $0.20 \mathrm{~ms}^{2}$; low-frequency/high-frequency ratio, $-0.173 \mathrm{vs.} 0.054$ ), and endurance exercise performance (maximal oxygen uptake, 5.57 vs. $2.26 \mathrm{~mL} / \mathrm{kg} / \mathrm{min} ; 400-\mathrm{m}$ time trial record, -20.41 vs. $-7.91 \mathrm{~s}$ ) than in the normoxia. These indicate that hypoxic training composed of aerobic continuous and interval exercise improves the endurance exercise performance of amateur male swimmers with better hemodynamic function and ANS function.
\end{abstract}

Keywords: aerobic continuous and interval training; amateur male swimmers; autonomic nervous system function; endurance exercise performance; hemodynamic function; hypoxia

\section{Introduction}

Endurance exercise performance is highly correlated with various physiological components that can be altered by diversiform training methods under hypoxia, including hematological changes such as erythropoiesis and nonhematological changes such as exercise economy, hemodynamic function, capillary density, and acid-base response in the skeletal muscle [1,2]. Accordingly, exercise training under hypoxia has been used worldwide for decades to enhance endurance exercise performance [3-5].

Hypoxic training can reinforce endurance exercise performance by using three training methods. Living high training high (LHTH) was the first hypoxic training design to living and training design at 1500-4000 $\mathrm{m}$ in the natural altitude conditions that improve hematological function, including erythropoiesis and oxygen delivery capacity [6,7]. 
However, LHTH has a major limitation in that it fails to achieve training of the same intensities (e.g., exercise speed and load) as training in a normoxic condition [8]. To overcome these shortcomings of LHTH, living high training low (LHTL), living high at 2000-3000 m and simultaneously training low at below $1500 \mathrm{~m}$, was developed by Dr. Benjamin Levine and James Stray-Gundersen of the United States in the early 1990s [8]. LHTL simultaneously offers athletes the beneficial effects of hematological function (e.g., improved erythropoiesis) and normoxic training (i.e., maintenance of training intensity) [8,9]. In recent years, living low training high (LLTH) has received the most attention from various athletes because it is generally associated with shorter exposure time to hypoxia (approximately three to five sessions per week of 1-3 h), less effort, less time, and lower cost than LHTH and LHTL $[1,10]$.

LLTH for exercise performance consists of various types of training methods such as continuous hypoxic training (CHT), interval hypoxic training (IHT), sprint interval training under hypoxia (SIH), repeated sprint training under hypoxia (RSH), resistance training under hypoxia (RTH), and voluntary hypoventilation at a low lung volume (VHL) [11]. Among these various LLTH methods (e.g., CHT, IHT, SIH, RSH, RTH, and VHL), CHT and IHT are most commonly used to enhance endurance exercise performance $[1,4,5,10]$. However, few studies have examined the effects of hypoxic training consisting of CHT and IHT on endurance exercise performance (especially swimming performance) versus continuous and interval training in normoxia. Short-term exposure to hypoxia with high-intensity exercise such as CHT, IHT, and CHT + IHT improves endurance exercise performance by enhancing metabolic function, hemodynamic function, and exercise economy $[1,5,10,12,13]$. However, some studies reported that CHT, IHT, and CHT + IHT did not enhance endurance exercise performance [14-17]. These conflicting results are because changes in autonomic nervous system (ANS) function, which are highly correlated with endurance exercise performance, have not been reviewed [10,18-20].

Heart rate variability (HRV) reflects the interaction between the sympathetic nervous system (SNS) and parasympathetic nervous system (PNS), which regulate cardiovascular function, and is an effective test method for quantitatively evaluating ANS activity and balance [21]. Dynamic modulation of cardiac and peripheral vascular regulation, including their regulation by the ANS, occurs in response to rapid changes in heart rate (HR) [21,22]. The improvement of HRV function via exercise training is often interpreted as enhanced ANS balance function or vagus nerve activity, which is related to endurance exercise performance $[23,24]$. Therefore, it is essential to verify the effect of exercise training under hypoxia on changes in HRV related to endurance exercise performance versus normoxia to verify the effectiveness of hypoxic training. However, studies to date of changes in ANS function following hypoxic training are scarce.

Therefore, the purpose of this study was to evaluate the effects of aerobic continuous and interval training under hypoxia on hemodynamic function, ANS function, and endurance exercise performance in amateur male swimmers. We hypothesized that aerobic continuous and interval training under hypoxia would improve endurance exercise performance by enhancing hemodynamic and ANS function in amateur male swimmers versus normoxia.

\section{Materials and Methods}

\subsection{Subjects}

The subjects were competitive amateur male swimmers $(n=20)$ with no experience in any exercise and training program in normobaric or hypobaric hypoxia (Table 1 ). They were equally assigned to the normoxic training group (NTG; $n=10$ ) or hypoxic training group (HTG; $n=10)$ according to endurance exercise performance and body composition. We explained the experiments and possible side effects to all amateur male swimmers prior to the start of the study and obtained signed consent for participation. The present study was approved by the Institutional Review Board of Konkuk University (7001355-201510- 
HR-090) in Korea and was conducted in accordance with the provisions of the Declaration of Helsinki.

Table 1. Subjects' characteristics.

\begin{tabular}{cccc}
\hline Variable & NTG $(\boldsymbol{n}=\mathbf{1 0})$ & HTG $(\boldsymbol{n}=\mathbf{1 0})$ & $\boldsymbol{p}$ Value \\
\hline Environmental condition & Normoxia $(760 \mathrm{mmHg})$ & Hypoxia $(526 \mathrm{mmHg})$ & - \\
$(\mathrm{mmHg})$ & $23.90 \pm 3.07$ & $24.00 \pm 3.06$ & 0.943 \\
Age $($ years $)$ & $176.3 \pm 5.69$ & $176.36 \pm 6.04$ & 0.988 \\
Height $(\mathrm{cm})$ & $69.89 \pm 13.40$ & $70.18 \pm 10.94$ & 0.958 \\
Weight $(\mathrm{kg})$ & $23.04 \pm 2.88$ & $23.05 \pm 1.60$ & 0.992 \\
BMI $\left(\mathrm{kg} / \mathrm{m}^{2}\right)$ & $23.24 \pm 3.88$ & $23.28 \pm 3.91$ & 0.982
\end{tabular}

Values are expressed as the mean \pm standard deviation. BMI, body mass index; HTG, hypoxic training group; NTG, normoxic training group.

\subsection{Study Design}

The present study design is illustrated in Figure 1. Twenty amateur male swimmers were equally divided into the NTG $(n=10$; aerobic continuous and interval training in a normoxia; $760 \mathrm{mmHg})$ and HTG $(n=10$; aerobic continuous and interval training in a hypobaric hypoxia; $526 \mathrm{mmHg}$; simulated altitude of $3000 \mathrm{~m}$ ). All testing and training sessions were performed in a $6.5 \mathrm{~m}$ wide $\times 7.5 \mathrm{~m}$ long $\times 3 \mathrm{~m}$ high hypobaric hypoxic chamber (Submersible Systems, Huntington Beach, CA, USA). The temperature within the hypobaric hypoxic chamber was maintained at $20 \pm 2{ }^{\circ} \mathrm{C}$, and the humidity was maintained at $60 \pm 2 \%$.

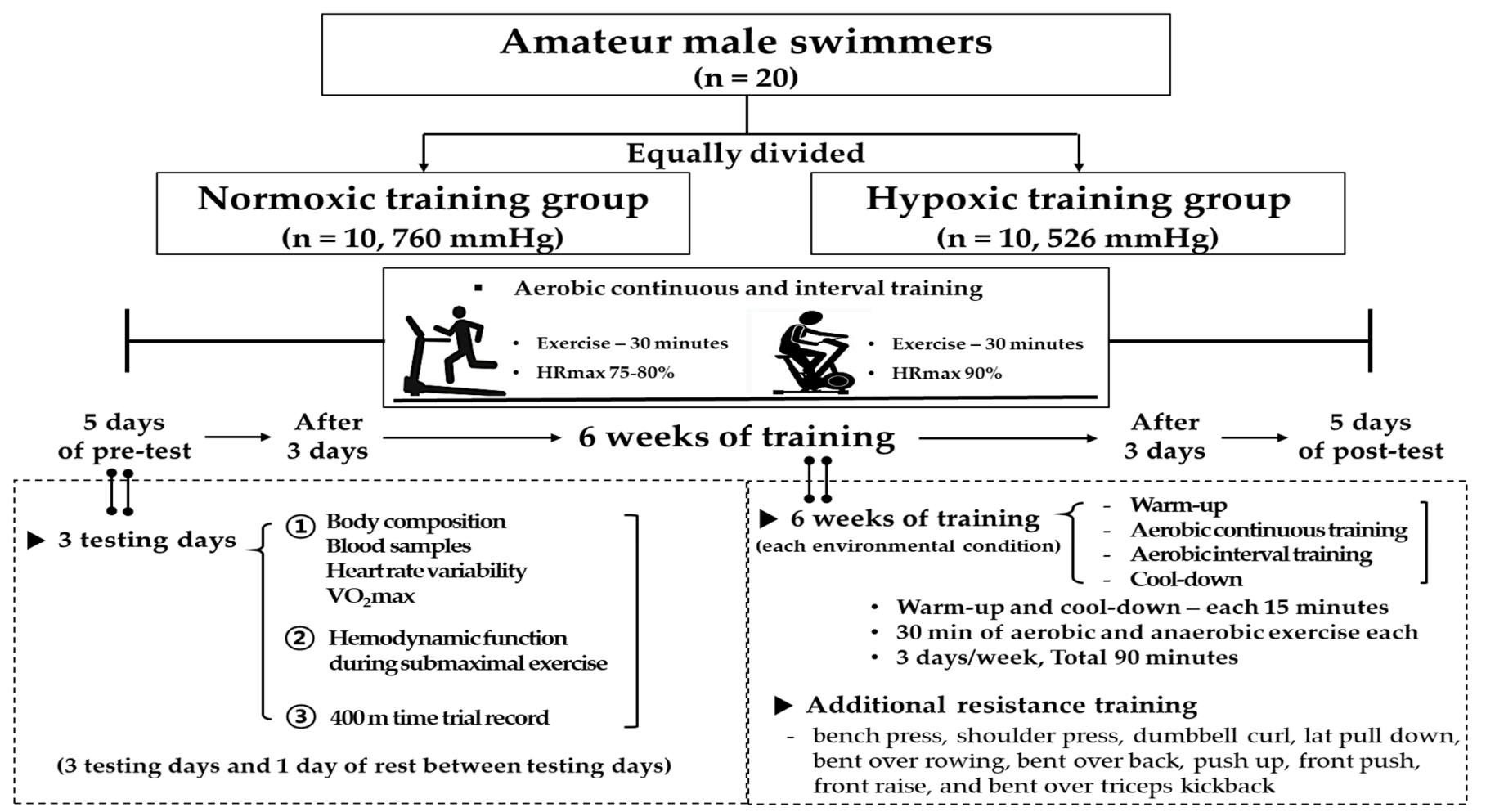

Figure 1. Study design. $\mathrm{HR}_{\max }$, maximal heart rate; $\mathrm{VO}_{2 \max }$, maximal oxygen uptake.

The experimental design consisted of the following: a 5-day pre-test period (i.e., 3 testing days and 1 rest day between them), a 6-week training period under each environmental condition, and a 5-day post-test period. The post-test period began 3 days after the final training session. During the 3 days of test sessions, body composition, hematological pa- 
rameters, hemodynamic function, ANS function, and endurance exercise performance were evaluated.

On the first pre- and post-testing days, venous blood samples were collected between 8:00 and 10:00 a.m. after $12 \mathrm{~h}$ of fasting to analyze hematological parameters and serum cortisol levels. Thereafter, the body composition and HRV parameters were measured. Subsequently, the maximal oxygen uptake $\left(\mathrm{VO}_{2 \max }\right)$ was measured to evaluate the endurance exercise performance in the afternoon. On the second pre- and post-testing days, hemodynamic function parameters were measured during a 30-min bout of submaximal cycle ergometer exercise. The exercise intensity was set at individual cycle ergometer exercise load values corresponding to $70 \%$ maximal $\mathrm{HR}\left(\mathrm{HR}_{\max }\right)$ obtained during the pre-test period. On the third testing day, a $400-\mathrm{m}$ time trial record in freestyle was measured by an automatic system installed on an authorized indoor swimming pool $(50 \mathrm{~m})$ at sea level in Suwon.

During the 6-week training period, 20 amateur male swimmers were equally divided into the NTG $(n=10)$ and HTG $(n=10)$, and they performed four kinds of training sessions in each environmental condition (NTG: normoxia, $760 \mathrm{mmHg}$; HTG: simulated $3000 \mathrm{~m}$ hypobaric hypoxia, $526 \mathrm{mmHg}$ ) for $90 \mathrm{~min}$ : warm-up, aerobic continuous exercise, aerobic interval exercise, and cool-down. The training frequency was $90 \mathrm{~min} 3$ days per week for 6 weeks. Warm-up and cool-down were set at 50\% maximum heart rate $\left(\mathrm{HR}_{\max }\right)$ for each subject for $5 \mathrm{~min}$, then increased by $10 \% \mathrm{HR}_{\max }$ every $5 \mathrm{~min}$ and performed for $15 \mathrm{~min}$. Continuous aerobic exercise was performed on a treadmill (Precor 932i, Precor, WA, USA) at $75 \% \mathrm{HR}_{\max }$ for $30 \mathrm{~min}$ and aerobic interval exercise on a cycle ergometer (Monark Exercise AB, Vansbro, Sweden) set at the exercise load with $90 \% \mathrm{HR}_{\max }$ measured in pre-test for $30 \mathrm{~min}(10 \times 2$-min exercise and 1-min rest). The velocity during warm-up, aerobic continuous exercise, and cool-down on a treadmill was changed using an HR monitor (Polar S610i, Helsinki, Finland) to match each HR. Anaerobic interval exercise intensity was set at individual bicycle exercise load values (watts) with $90 \% \mathrm{HR}_{\max }$ obtained at pre-test in each environmental condition.

In addition, all participants performed equally additional resistance training sessions (three sets of 8-10 repetitions at an exercise intensity range of $70-80 \%$ of one-repetition maximum, with 60-s rest per set) in a normoxia composed of a bench press, shoulder press, dumbbell curl, lat pull-down, bent-over-rowing, bent-over-back, push-up, front push, front raise, and bent-over tricep kickback. Additional resistance training frequency was 60 min 3 days per week for 6 weeks.

All exercise training sessions in normoxia or hypoxia were supervised by researchers, coaches, and directors.

\subsection{Body Composition}

Body composition parameters (e.g., weight, body mass index, and \% body fat) were evaluated after $12 \mathrm{~h}$ of fasting using bioelectrical impedance analysis. All participants wore lightweight clothing and were asked to remove any metal items. An Inbody 770 device (Inbody, Seoul, Korea) was used to measure body composition.

\subsection{Hematological Parameters}

Venous blood samples were performed on pre- and post-testing days at rest in normoxia to measure hematological parameters. A 5-mL sample of venous blood samples was collected in a heparin tube for whole blood (3-mL) and a serum separation tube for serum (2-mL).

To analyze hematological parameters, blood samples were obtained before and after training at rest at normoxia. A 5-mL sample of venous blood was collected in a heparin tube for whole blood (3-mL) and a serum separation tube for serum (2-mL). An XE2100D hematology analyzer (Sysmex, Kobe, Japan) was used to analyze the red blood cell (RBC) count, hemoglobin $(\mathrm{Hb})$ concentration, and hematocrit (Hct). The RBC count and Hct were measured using an impedance-based method. $\mathrm{Hb}$ concentration was measured using 
cyanide-free $\mathrm{Hb}$ spectrophotometry. Erythropoietin (EPO) levels were measured using an Immulite 2000 XPI analyzer (Siemens, Eschborn, Germany) using the chemiluminescent immunoassay method. Mean corpuscular volume (MCV), mean corpuscular hemoglobin $(\mathrm{MCH})$, and mean corpuscular hemoglobin concentration $(\mathrm{MCHC})$ were calculated using the following formulas: $\mathrm{MCV}=($ Hct/erythrocyte $) \times 10 ; \mathrm{MCH}=(\mathrm{Hb} /$ erythrocyte $) \times 10$; and $\mathrm{MCHC}=(\mathrm{Hb} / \mathrm{Hct}) \times 100$.

\subsection{Hemodynamic Function Parameters}

Hemodynamic function parameters were evaluated on pre- and post-testing days. All subjects performed a submaximal exercise using a cycle ergometer with a load corresponding to $75 \% \mathrm{HRmax}$ measured before training in normoxia. Oxygen uptake $\left(\mathrm{VO}_{2}\right)$ was measured using a Vmax-229 breath-by-breath auto metabolism analyzer (SensorMedics, Yorba Linda, CA, USA). HR, stroke volume index (SVi), and cardiac output index (COi) were evaluated noninvasively using a thoracic bioelectrical impedance device (PhysioFlow PF-05, Paris, France). All variables were measured every minute, and the total values were used for $\mathrm{HR}, \mathrm{VO}_{2}, \mathrm{SVi}$, and $\mathrm{CO}$.

\subsection{ANS Function}

ANS function was assessed by measuring HRV and serum cortisol levels. After approximately 10 min of rest, four pads were placed on the wrists and ankles using an HRV meter (LAXTHA; CANS-3000, Daejeon, Korea), and all male amateur swimmers' HRV was evaluated in the resting condition. The following parameters were measured: standard deviation of successive differences (SDNN) and root mean square of successive differences (RMSSD) for the time domain methods and total power (TP), low-frequency (LF), high-frequency (HF), and LH/HF ratio for the frequency domain methods. Serum cortisol levels were determined using radioimmunoassay (Coat-A-count; Siemens, Eschborn, Germany).

\subsection{Endurance Exercise Performance}

To evaluate exercise performance, $\mathrm{VO}_{2 \max }$ was measured before and after training using the BRUCE protocol for graded exercise testing on a treadmill (Precor 932i) with a Vmax-229 breath-by-breath auto metabolism analyzer (SensorMedics) under normoxia. The 400-m time trial records in freestyle were measured twice by an automatic system installed on an authorized indoor swimming pool $(50 \mathrm{~m})$ at sea level in Suwon, and the average time was used.

\subsection{Statistical Analysis}

All statistical analyses were conducted using SPSS version 25.0 (IBM Corp., Armonk, NY, USA) for Windows. Data are presented as mean \pm standard deviation. The normality of the distribution of all outcome variables was verified using the Shapiro-Wilk test. A two-way analysis (time $\times$ group) of variance with repeated measures of the "time" factor was used to analyze the effects of training programs on each dependent variable. Partial eta-squared $\left(\eta^{2}\right)$ values were calculated as measures of the effect size. If a significant interaction effect was found, a Bonferroni post hoc test was used to identify intragroup changes over time. Additionally, the paired t-test was used to compare the pre- and posttraining values of dependent variables in each group separately. An a priori power analysis was performed with G-power for the hemodynamic function parameter $\left(\mathrm{VO}_{2}\right.$ during submaximal exercise) based on previous research [1], indicating that a sample size of 16 participants ( 8 participants per group) would be required to provide $88 \%$ power at an $\alpha$-level of 0.05 . We anticipated a more than $10 \%$ dropout rate and aimed for a starting population of 20. The level of significance was set a priori at $p<0.05$. 


\section{Results \\ 3.1. Body Composition}

Table 2 shows the body composition parameters before and after training in both groups. No significant interaction was observed in any of the body composition parameters, that is, body composition did not affect changes in the other dependent parameters.

Table 2. Changes in body composition before and after training by group.

\begin{tabular}{|c|c|c|c|c|c|c|c|c|c|}
\hline \multirow[b]{2}{*}{ Measures } & \multicolumn{3}{|c|}{ NTG $(n=10)$} & \multicolumn{3}{|c|}{ HTG $(n=10)$} & \multicolumn{3}{|c|}{$\eta^{2}(p)$ Value } \\
\hline & Before & After & $\begin{array}{l}\text { Mean Change } \\
(95 \% \text { CI })\end{array}$ & Before & After & $\begin{array}{l}\text { Mean Change } \\
(95 \% \text { CI })\end{array}$ & Time & Group & Interaction \\
\hline Weight (kg) & $69.89 \pm 13.40$ & $69.30 \pm 13.24$ & $\begin{array}{c}-0.60 \\
(-1.43,0.24)\end{array}$ & $70.18 \pm 10.94$ & $70.01 \pm 11.34$ & $\begin{array}{c}-0.17 \\
(-1.08,0.74)\end{array}$ & $\begin{array}{c}0.098 \\
(0.179)\end{array}$ & $\begin{array}{c}0.000 \\
(0.928)\end{array}$ & $\begin{array}{c}0.032 \\
(0.447)\end{array}$ \\
\hline BMI $\left(\mathrm{kg} / \mathrm{m}^{2}\right)$ & $23.04 \pm 2.88$ & $22.58 \pm 2.33$ & $\begin{array}{c}-0.46 \\
(-1.54,0.59)\end{array}$ & $23.05 \pm 1.60$ & $22.44 \pm 1.58$ & $\begin{array}{c}-0.61 \\
(-1.00,-0.22)\end{array}$ & $\begin{array}{c}0.205 \\
(0.045)+\end{array}$ & $\begin{array}{c}0.000 \\
(0.945)\end{array}$ & $\begin{array}{c}0.005 \\
(0.766)\end{array}$ \\
\hline Percent body fat (\%) & $23.24 \pm 3.88$ & $23.18 \pm 3.65$ & $\begin{array}{c}-0.06 \\
(-1.15,1.03)\end{array}$ & $23.18 \pm 3.65$ & $22.57 \pm 3.90$ & $\begin{array}{c}-0.71 \\
(-1.87,0.45)\end{array}$ & $\begin{array}{c}0.062 \\
(0.290)\end{array}$ & $\begin{array}{c}0.002 \\
(0.867)\end{array}$ & $\begin{array}{c}0.045 \\
(0.370)\end{array}$ \\
\hline
\end{tabular}

Values are expressed as mean \pm standard deviation. BMI, body mass index; CI, confidence interval; HTG, hypoxic training group; NTG, normoxic training group. Significant interaction or main effect: $\dagger p<0.05$.

\subsection{Hematological Parameters}

As shown in Table 3, there was a significant interaction between $\operatorname{RBC}\left(\eta^{2}=0.417\right.$, $p=0.002), \mathrm{Hb}\left(\eta^{2}=0.397, p=0.003\right)$, and Hct $\left(\eta^{2}=0.479, p=0.001\right)$. Post hoc analyses showed that both groups showed a significant decrease in RBC (HTG $-0.32,95 \%$ CI $-0.39,-0.24$; NTG $-0.14,95 \%$ CI $-0.22,-0.05106 / \mathrm{uL}, p<0.05)$ and $\mathrm{Hb}(\mathrm{HTG}-0.87$, $95 \%$ CI $-1.11,-0.63$; NTG $-0.38,95 \%$ CI $-0.60,-0.15 \mathrm{~kg}, p<0.05)$, but HTG showed a greater decrease than NTG. Hct (HTG $-2.41,95 \%$ CI $-3.12,-1.70 \mathrm{~kg}, p<0.05)$ presented a significant decrease only in HTG. No significant interaction was observed with EPO, MCV, $\mathrm{MCH}$, and $\mathrm{MCHC}$.

Table 3. Changes in hematological parameters before versus after training by group.

\begin{tabular}{|c|c|c|c|c|c|c|c|c|c|}
\hline \multirow[b]{2}{*}{ Measures } & \multicolumn{3}{|c|}{ NTG $(n=10)$} & \multicolumn{3}{|c|}{ HTG $(n=10)$} & \multicolumn{3}{|c|}{$\eta^{2}(p)$ Value } \\
\hline & Before & After & $\begin{array}{c}\text { Mean Change } \\
(95 \% \mathrm{CI})\end{array}$ & Before & After & $\begin{array}{c}\text { Mean Change } \\
(95 \% \mathrm{CI})\end{array}$ & Time & Group & Interaction \\
\hline $\mathrm{RBC}\left(\times 10^{6} / \mu \mathrm{L}\right)$ & $4.70 \pm 0.49$ & $4.57 \pm 0.50$ & $\begin{array}{c}-0.14^{*} \\
(-0.22,-0.05)\end{array}$ & $5.16 \pm 0.44$ & $4.84 \pm 0.43$ & $\begin{array}{c}-0.32 * \\
(-0.39,-0.24)\end{array}$ & $\begin{array}{c}0.820 \\
(0.000)+\end{array}$ & $\begin{array}{c}0.144 \\
(0.099)\end{array}$ & $\begin{array}{c}0.417 \\
(0.002)+\end{array}$ \\
\hline $\mathrm{Hb}(\mathrm{g} / \mathrm{dL})$ & $14.79 \pm 1.43$ & $14.41 \pm 1.46$ & $\begin{array}{c}-0.38 * \\
(-0.60,-0.15)\end{array}$ & $15.51 \pm 1.21$ & $14.64 \pm 1.31$ & $\begin{array}{c}-0.87 * \\
(-1.11,-0.63)\end{array}$ & $\begin{array}{c}0.806 \\
(0.000)+\end{array}$ & $\begin{array}{c}0.033 \\
(0.813)\end{array}$ & $\begin{array}{c}0.397 \\
(0.003) \dagger\end{array}$ \\
\hline Hct (\%) & $44.35 \pm 3.41$ & $43.95 \pm 3.73$ & $\begin{array}{c}-0.40 \\
(-1.29,0.49)\end{array}$ & $46.40 \pm 3.19$ & $43.99 \pm 3.46$ & $\begin{array}{c}-2.41 * \\
(-3.12,-1.70)\end{array}$ & $\begin{array}{c}0.642 \\
(0.000)+\end{array}$ & $\begin{array}{c}0.025 \\
(0.502)\end{array}$ & $\begin{array}{c}0.479 \\
(0.001) \dagger\end{array}$ \\
\hline $\mathrm{EPO}(\mathrm{mU} / \mathrm{mL})$ & $9.11 \pm 2.22$ & $10.16 \pm 3.47$ & $\begin{array}{c}2.30 \\
(0.87,3.72)\end{array}$ & $10.16 \pm 3.47$ & $15.25 \pm 2.00$ & $\begin{array}{c}5.09 \\
(2.16,8.01)\end{array}$ & $\begin{array}{c}0.593 \\
(0.000)+\end{array}$ & $\begin{array}{c}0.271 \\
(0.019) \dagger\end{array}$ & $\begin{array}{c}0.173 \\
(0.068)\end{array}$ \\
\hline $\operatorname{MCV}\left(\mu \mathrm{m}^{2}\right)$ & $94.58 \pm 3.52$ & $96.54 \pm 3.40$ & $\begin{array}{c}1.95 \\
(1.59,2.31)\end{array}$ & $90.15 \pm 2.90$ & $91.03 \pm 2.16$ & $\begin{array}{c}0.88 \\
(-0.73,2.49)\end{array}$ & $\begin{array}{c}0.456 \\
(0.001)+\end{array}$ & $\begin{array}{c}0.444 \\
(0.001) \dagger\end{array}$ & $\begin{array}{c}0.107 \\
(0.159)\end{array}$ \\
\hline $\mathrm{MCH}(\mu \mathrm{g})$ & $31.49 \pm 1.17$ & $31.61 \pm 1.04$ & $\begin{array}{c}0.12 \\
(-0.11,0.36)\end{array}$ & $30.14 \pm 1.32$ & $30.29 \pm 1.30$ & $\begin{array}{c}0.16 \\
(-0.14,0.46)\end{array}$ & $\begin{array}{c}0.135 \\
(0.111)\end{array}$ & $\begin{array}{c}0.258 \\
(0.022) \dagger\end{array}$ & $\begin{array}{c}0.003 \\
(0.823)\end{array}$ \\
\hline $\mathrm{MCHC}(\mathrm{g} / \mathrm{dL})$ & $33.30 \pm 0.84$ & $32.75 \pm 0.63$ & $\begin{array}{c}-0.55 \\
(-0.84,-0.26)\end{array}$ & $33.42 \pm 0.91$ & $33.27 \pm 0.96$ & $\begin{array}{c}-0.15 \\
(-0.63,0.33)\end{array}$ & $\begin{array}{c}0.310 \\
(0.011)+\end{array}$ & $\begin{array}{c}0.042 \\
(0.395)\end{array}$ & $\begin{array}{c}0.128 \\
(0.122)\end{array}$ \\
\hline
\end{tabular}

Values are expressed as means \pm standard deviations. CI, confidence interval; EPO, erythropoietin; Hb, hemoglobin; Hct, hematocrit; HTG, hypoxic training group; $\mathrm{MCV}$, mean corpuscular volume; $\mathrm{MCH}$, mean corpuscular hemoglobin; $\mathrm{MCHC}$, mean corpuscular hemoglobin concentration; NTG, normoxic training group; RBC, red blood cells. Significant interaction or main effect: $\uparrow p<0.05$; Significant difference before and after training: ${ }^{*} p<0.05$.

\subsection{Hemodynamic Function}

As shown in Table 4, there was a significant interaction between $\operatorname{HR}\left(\eta^{2}=0.256, p=0.023\right)$, $\mathrm{VO}_{2}\left(\eta^{2}=0.237, p=0.030\right)$, and SVi $\left(\eta^{2}=0.308, p=0.011\right)$. Post hoc analyses found significant decrease in both groups for HR (HTG, $-653.4 ; 95 \%$ CI, -795.4 to -511.4 beats $/ 30$ min; NTG, $-353.7 ; 95 \%$ CI, -586.1 to -121.3 beats $/ 30 \mathrm{~min} ; p<0.05$ ) and SVi (HTG, 197.66; 95\% CI, 90.10-305.22 mL/30 min; NTG, 52.32; 95\% CI, 8.54-96.10 mL/30 min; $p<0.05)$. 
$\mathrm{VO}_{2}$ (HTG, $-62.45 ; 95 \% \mathrm{CI},-91.16$ to $\left.-33.74 \mathrm{~mL} / \mathrm{kg} / 30 \mathrm{~min} ; p<0.05\right)$ showed a significant decrease in the HTG only. However, no significant interaction was observed for COi. Overall, hemodynamic function improved more in the HTG than in the NTG.

Table 4. Changes in hemodynamic function during submaximal exercise for $30 \mathrm{~min}$ before and after training by group.

\begin{tabular}{|c|c|c|c|c|c|c|c|c|c|}
\hline \multirow[b]{2}{*}{ Measure } & \multicolumn{3}{|c|}{ NTG $(n=10)$} & \multicolumn{3}{|c|}{ HTG $(n=10)$} & \multicolumn{3}{|c|}{$\eta^{2}(p)$ Value } \\
\hline & Before & After & $\begin{array}{c}\text { Mean Change } \\
(95 \% \mathrm{CI})\end{array}$ & Before & After & $\begin{array}{l}\text { Mean Change } \\
(95 \% \mathrm{CI})\end{array}$ & Time & Group & Interaction \\
\hline $\begin{array}{c}\text { HR } \\
\text { (beats } / 30 \mathrm{~min} \text { ) }\end{array}$ & $\begin{array}{c}4980.3 \\
\pm 420.0\end{array}$ & $\begin{array}{l}4626.6 \\
\pm 210.0\end{array}$ & $\begin{array}{c}-353.7 \\
(-586.1 \\
-121.3)^{*}\end{array}$ & $\begin{array}{l}4911.3 \\
\pm 218.6\end{array}$ & $\begin{array}{c}4257.9 \\
\pm 163.7\end{array}$ & $\begin{array}{c}-653.4 \\
(-795.4 \\
-511.4)^{*}\end{array}$ & $\begin{array}{c}0.795 \\
(0.000)+\end{array}$ & $\begin{array}{c}0.193 \\
(0.053)\end{array}$ & $\begin{array}{c}0.256 \\
(0.023)+\end{array}$ \\
\hline $\begin{array}{c}\mathrm{VO}_{2} \\
(\mathrm{~mL} / \mathrm{kg} / 30 \mathrm{~min})\end{array}$ & $\begin{array}{l}1130.4 \\
\pm 192.1\end{array}$ & $\begin{array}{c}1114.2 \\
\pm 173.4\end{array}$ & $\begin{array}{c}-16.22 \\
(-49.92,17.47)\end{array}$ & $\begin{array}{c}1115.8 \\
\pm 161.6\end{array}$ & $\begin{array}{l}1053.3 \\
\pm 152.6\end{array}$ & $\begin{array}{l}-62.45 \\
(-91.16 \\
-33.74)^{*}\end{array}$ & $\begin{array}{c}0.473 \\
(0.001)+\end{array}$ & $\begin{array}{c}0.014 \\
(0.624)\end{array}$ & $\begin{array}{c}0.237 \\
(0.030)+\end{array}$ \\
\hline $\begin{array}{c}\mathrm{SVi} \\
(\mathrm{mL} / 30 \mathrm{~min})\end{array}$ & $\begin{array}{l}1497.8 \\
\pm 98.8\end{array}$ & $\begin{array}{c}1550.1 \\
\pm 100.4\end{array}$ & $\begin{array}{c}52.32 \\
(8.54,96.10) *\end{array}$ & $\begin{array}{c}1514.3 \\
\pm 194.0\end{array}$ & $\begin{array}{c}1712.0 \\
\pm 110.6\end{array}$ & $\begin{array}{c}197.66 \\
(90.10 \\
305.22) *\end{array}$ & $\begin{array}{c}0.568 \\
(0.000)+\end{array}$ & $\begin{array}{c}0.135 \\
(0.111)\end{array}$ & $\begin{array}{c}0.308 \\
(0.011)+\end{array}$ \\
\hline $\begin{array}{c}\text { COi } \\
(\mathrm{L} / 30 \mathrm{~min})\end{array}$ & $\begin{array}{c}240.9 \\
\pm 18.5\end{array}$ & $\begin{array}{c}224.9 \\
\pm 20.5\end{array}$ & $\begin{array}{c}-15.95 \\
(-41.72,9.83)\end{array}$ & $\begin{array}{l}251.8 \\
\pm 31.2\end{array}$ & $\begin{array}{l}227.6 \\
\pm 34.2\end{array}$ & $\begin{array}{c}-24.25 \\
(-57.80,9.30)\end{array}$ & $\begin{array}{c}0.204 \\
(0.045) \dagger\end{array}$ & $\begin{array}{c}0.043 \\
(0.382)\end{array}$ & $\begin{array}{c}0.011 \\
(0.662)\end{array}$ \\
\hline
\end{tabular}

Values are expressed as means \pm standard deviations. CI, confidence interval; $\mathrm{CO}$, cardiac output index; HTG, hypoxic training group; $\mathrm{HR}$, heart rate; NTG, normoxic training group; $\mathrm{SVi}$, stroke volume index; $\mathrm{VO}_{2}$, oxygen uptake. Significant interaction or main effect: $+p<0.05$; Significant difference before versus after training: ${ }^{*} p<0.05$.

\subsection{ANS Function}

Table 5 presents a significant interaction in $\operatorname{RMSSD}\left(\eta^{2}=0.227, p=0.034\right), \operatorname{TP}\left(\eta^{2}=0.405\right.$, $p=0.003)$, and LF/HF ratio $\left(\eta^{2}=0.226, p=0.034\right)$. Post hoc analyses revealed that the HTG had a significantly improved mean RMSSD (HTG, 10.15; 95\% CI, 4.37-15.94 $\mathrm{ms}^{2}$; $p<0.05$ ), TP (HTG, $0.72 ; 95 \% \mathrm{CI}, 0.50-0.94 \mathrm{~ms}^{2} ; p<0.05$ ), and LF/HF ratio (HTG, -0.173 ; $95 \%$ CI,-0.277 to $-0.069 ; p<0.05)$. However, no significant interaction was observed among SDNN, LF, HF, and serum cortisol levels.

Table 5. Changes in autonomic nervous system function before versus after training by group.

\begin{tabular}{|c|c|c|c|c|c|c|c|c|c|}
\hline \multirow[b]{2}{*}{ Measures } & \multicolumn{3}{|c|}{ NTG $(n=10)$} & \multicolumn{3}{|c|}{ HTG $(n=10)$} & \multicolumn{3}{|c|}{$\eta^{2}(p)$ Value } \\
\hline & Before & After & $\begin{array}{c}\text { Mean Change } \\
(95 \% \mathrm{CI})\end{array}$ & Before & After & $\begin{array}{c}\text { Mean Change } \\
(95 \% \mathrm{CI})\end{array}$ & Time & Group & Interaction \\
\hline SDNN (ms) & $54.06 \pm 6.06$ & $55.11 \pm 6.60$ & $\begin{array}{c}1.04 \\
(-4.06,6.15)\end{array}$ & $52.91 \pm 11.54$ & $59.16 \pm 9.83$ & $\begin{array}{c}6.25 \\
(-0.39,12.1)\end{array}$ & $\begin{array}{c}0.200 \\
(0.048)+\end{array}$ & $\begin{array}{c}0.009 \\
(0.688)\end{array}$ & $\begin{array}{c}0.113 \\
(0.147)\end{array}$ \\
\hline RMSSD (ms) & $32.18 \pm 7.91$ & $35.50 \pm 8.81$ & $\begin{array}{c}3.32 \\
(-0.10,6.74)\end{array}$ & $31.18 \pm 8.31$ & $41.33 \pm 9.60$ & $\begin{array}{c}10.15 \\
(4.37,15.94) *\end{array}$ & $\begin{array}{c}0.533 \\
(0.000)+\end{array}$ & $\begin{array}{c}0.025 \\
(0.509)\end{array}$ & $\begin{array}{c}0.227 \\
(0.034)+\end{array}$ \\
\hline $\mathrm{TP}\left(\mathrm{ms}^{2}\right)$ & $8.04 \pm 0.22$ & $8.24 \pm 0.26$ & $\begin{array}{c}0.20 \\
(-0.05,0.46)\end{array}$ & $7.75 \pm 0.47$ & $8.47 \pm 0.40$ & $\begin{array}{c}0.72 \\
(0.50,0.94) *\end{array}$ & $\begin{array}{c}0.685 \\
(0.000)+\end{array}$ & $\begin{array}{c}0.002 \\
(0.843)\end{array}$ & $\begin{array}{c}0.405 \\
(0.003)+\end{array}$ \\
\hline $\mathrm{LF}\left(\mathrm{ms}^{2}\right)$ & $6.64 \pm 0.48$ & $6.46 \pm 0.36$ & $\begin{array}{c}-0.18 \\
(-0.43,0.63)\end{array}$ & $6.62 \pm 0.49$ & $6.30 \pm 0.59$ & $\begin{array}{c}-0.32 \\
(-0.48,-0.17)\end{array}$ & $\begin{array}{c}0.463 \\
(0.001)+\end{array}$ & $\begin{array}{c}0.011 \\
(0.661)\end{array}$ & $\begin{array}{c}0.062 \\
(0.288)\end{array}$ \\
\hline $\mathrm{HF}\left(\mathrm{ms}^{2}\right)$ & $6.40 \pm 0.38$ & $6.56 \pm 0.49$ & $\begin{array}{c}0.17 \\
(-0.13,0.46)\end{array}$ & $6.01 \pm 0.89$ & $6.69 \pm 0.65$ & $\begin{array}{c}0.69 \\
(0.20,1.17)\end{array}$ & $\begin{array}{c}0.392 \\
(0.003)+\end{array}$ & $\begin{array}{c}0.015 \\
(0.611)\end{array}$ & $\begin{array}{c}0.192 \\
(0.053)\end{array}$ \\
\hline LF/HF ratio & $1.04 \pm 0.071$ & $0.986 \pm 0.048$ & $\begin{array}{c}0.054 \\
(-0.109,0.001)\end{array}$ & $1.12 \pm 0.178$ & $0.948 \pm 0.127$ & $\begin{array}{c}-0.173 \\
(-0.277,-0.069) \text { * }\end{array}$ & $\begin{array}{c}0.516 \\
(0.000)+\end{array}$ & $\begin{array}{c}0.012 \\
(0.644)\end{array}$ & $\begin{array}{c}0.226 \\
(0.034)+\end{array}$ \\
\hline $\begin{array}{l}\text { Serum cortisol } \\
(\mu \mathrm{g} / \mathrm{dL})\end{array}$ & $14.41 \pm 5.23$ & $12.97 \pm 3.75$ & $\begin{array}{c}-1.44 \\
(-4.50,1.62)\end{array}$ & $14.29 \pm 1.63$ & $10.93 \pm 2.37$ & $\begin{array}{c}-3.36 \\
(-5.48,-1.24)\end{array}$ & $\begin{array}{c}0.320 \\
(0.009)+\end{array}$ & $\begin{array}{c}0.035 \\
(0.430)\end{array}$ & $\begin{array}{c}0.070 \\
(0.258)\end{array}$ \\
\hline
\end{tabular}

Values are expressed as mean \pm standard deviation. CI, confidence interval; HF, high-frequency; HTG, hypoxic training group; LF, low-frequency; NTG, normoxic training group; RMSSD, root mean square of successive differences; SDNN, standard deviation of NN intervals; TP, total power. Significant interaction or main effect: $\dagger p<0.05$; Significant difference before versus after training: ${ }^{*} p<0.05$.

\subsection{Endurance Exercise Performance}

Figure 2 depicts the body composition parameters before and after training by group. There was a significant interaction between $\mathrm{VO}_{2 \max }\left(\eta^{2}=0.406, p=0.002\right)$ and a 400-m time trial record $\left(\eta^{2}=0.663, p<0.001\right)$. Post hoc analyses revealed a significantly improved $\mathrm{VO}_{2 \max }$ (HTG, 5.57; 95\% CI, 4.19-7.35; NTG, 2.26; 95\% CI, 0.64-3.88 mL $/ \mathrm{kg} / \mathrm{min} ; p<0.05$ ) and $400-\mathrm{m}$ time trial record (HTG, $-20.41 ; 95 \% \mathrm{CI},-23.68$ to $-17.14 ; \mathrm{NTG},-7.91 ; 95 \% \mathrm{CI}$, 
-11.36 to $-4.46 \mathrm{~s} ; p<0.05)$ in both groups. Consequently, endurance exercise performance induced greater improvement in the HTG than in the NTG.
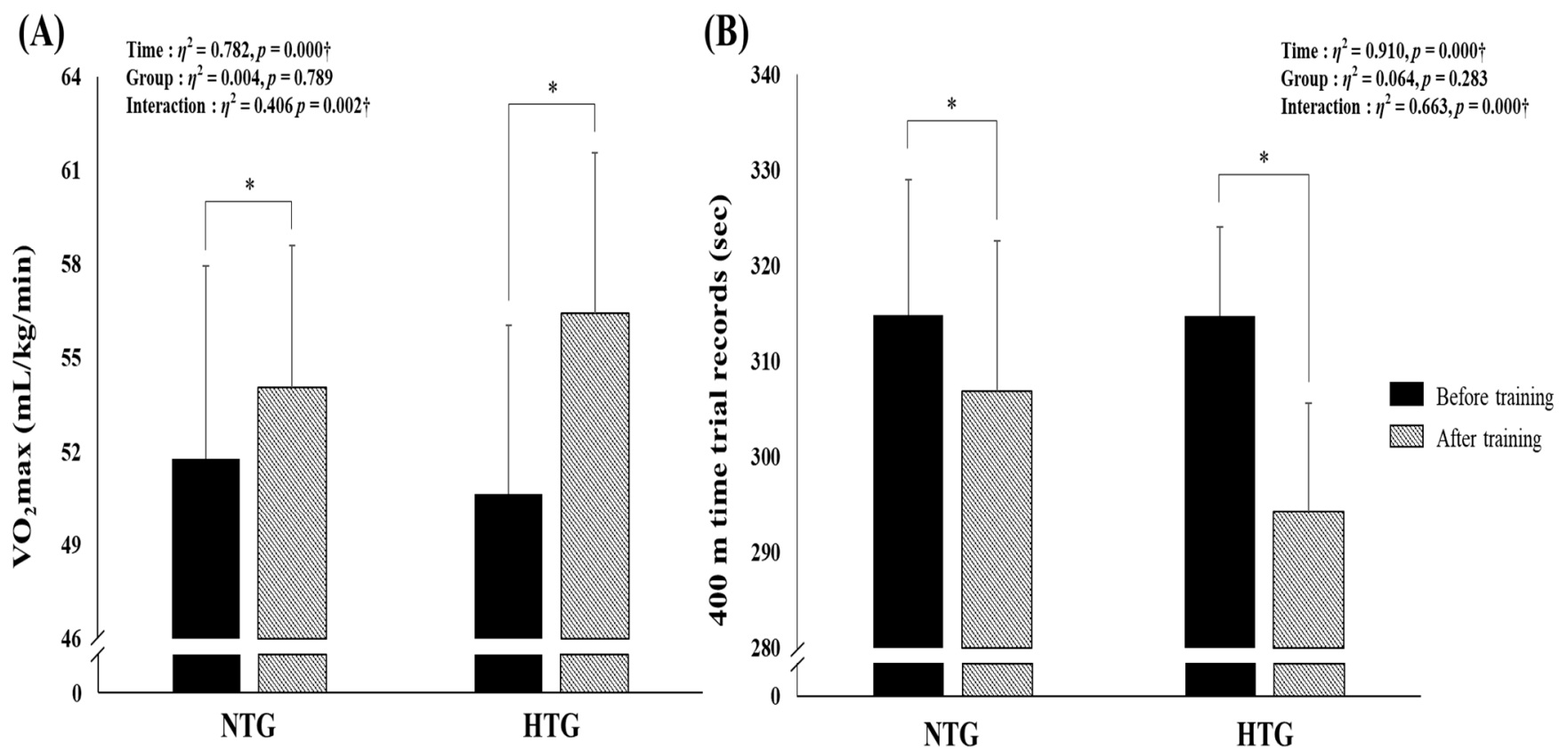

Figure 2. Changes in endurance exercise performance parameters before versus after training by group. (A) Change in $\mathrm{VO}_{2 \mathrm{max}}$ before versus after exercise training in each environmental condition. (B) Change in the 400-m time trial records before versus after exercise training in each environmental condition. HTG, hypoxic training group; NTG, normoxic training group; $\mathrm{VO}_{2 \max }$, maximum oxygen uptake. Significant interaction or main effect: $+p<0.05$; Significant difference before versus after training: ${ }^{*} p<0.05$.

\section{Discussion}

In our study, we hypothesized that aerobic continuous and interval training under hypoxia (simulated $3000 \mathrm{~m}, 526 \mathrm{mmHg}$ hypobaric hypoxia) would improve endurance exercise performance with enhanced hemodynamic and ANS function more than under normoxia in amateur male swimmers. The present study aimed to demonstrate that our hypoxic training program can sufficiently elicit a meaningful increase in endurance exercise performance with enhanced hemodynamic and ANS function in amateur male swimmers. Our findings are consistent with these hypotheses.

In general, exposure to hypoxia reportedly stimulates erythropoiesis to enhance the oxygen delivery capacity in people living under normoxia [25,26]. Erythropoiesis caused by hypoxia involves an increase in the mass of $\mathrm{Hb}$, a metal protein that carries oxygen in the RBC, and an increased RBC count [27]. In addition, EPO has the greatest influence on hematological changes caused by hypoxia [9]. When exposed to hypoxia, the EPO concentration acutely increases and peaks at approximately $48-72 \mathrm{~h}$, and the production of new by EPO stimulation reportedly takes nearly five days $[25,26,28]$. An increase in erythropoiesis and a decrease in plasma volume due to exposure to hypoxia means that the ability to deliver oxygen per unit of blood increases, but a decrease in plasma volume can adversely affect the oxygen delivery capacity through the circulatory system. In practice, endurance exercise performance in athletes is largely determined by the decrease in Hct and blood viscosity according to plasma volume $[9,29]$. However, exercise training under hypoxia such as LHTH and LHTL is very effective at improving blood oxygen delivery capacity because it induces an increase in plasma volume as well as an increase in RBC production via $\mathrm{EPO}$, and these effects were maintained for approximately 16 days after exposure to hypoxia $[26,29,30]$. 
The aerobic continuous and interval training under hypoxia used in our study is an LLTH (residing in normoxia but training under hypoxia), which does not induce an increase in hematological changes due to insufficient hypoxia stimulation because the exposure time to hypoxia is substantially less than $2 \mathrm{~h}$ per day [29,31]. In our study, six weeks of aerobic continuous and interval training under hypoxia did not show increased erythropoiesis, examined via hematological changes compared with normoxia. RBC count (HTG, $-0.32 \times 10^{6} / \mu \mathrm{L}$ vs. NTG $-0.14 \times 10^{6} / \mu \mathrm{L}$ ) and $\mathrm{Hb}$ concentration (HTG, $-0.87 \mathrm{~g} / \mathrm{dL}$ vs. NTG, $-0.38 \mathrm{~g} / \mathrm{dL}$ ) decreased significantly in both groups, and the reduction was greater in the HTG than in the NTG. However, Hct showed a significant decrease only in the HTG $(-2.41 \%)$. In other words, the greater decrease in RBC count and $\mathrm{Hb}$ concentration presented in the NTG is thought to be due to a greater increase in plasma volume by hypoxic versus normoxic training. Aerobic continuous and interval training under hypoxia confirmed the possibility of improving the oxygen delivery capacity by inducing a decrease in Hct via increasing plasma volume.

Hemodynamic function refers to the dynamics of blood flow in systemic conditions, and the hemodynamic system plays a role in distributing blood flow via continuous monitoring and adjustment of the conditions in the body and its environment [10]. In particular, hemodynamic function during submaximal exercise is closely related to endurance exercise performance as an indicator of oxygen delivery and use capacity $[1,10,12]$.

Hypoxic training such as CHT, IHT, and CHT + IHT effectively improved endurance exercise performance via increasing glycolysis enzyme activity, glucose delivery capacity, mitochondrial density, capillary density, acid-base balance regulation, cross-sectional area of skeletal muscle, and activity of the motor unit by stimulating the neuromuscular system $[1,5,13,29]$. These physiological, biochemical, and structural adaptative changes improve the efficiency of oxygen blood delivery and use capacity, thereby enhancing endurance exercise performance $[1,5,10,13,32]$. In addition, endurance exercise performance is improved by enhancing the exercise economy (defined as the amount of energy per unit distance), which represents the rate of oxygen flow into the skeletal muscle tissue and the ability of mitochondria to utilize oxygen $[1,9,33]$. Previous studies reported that a greater exercise economy is related to improved endurance exercise performance by physiological adaptations to hypoxic training $[1,9,10,34]$. However, there is a lack of previous studies that prove that hypoxic training such as $\mathrm{CHT}$, IHT, and $\mathrm{CHT}+\mathrm{IHT}$ improves endurance exercise performance based on hemodynamic function, which indicates oxygen delivery and utilization capacity in systemic conditions, including exercise economy. Therefore, we verified the effects of aerobic continuous and interval training under hypoxia on endurance exercise performance in relation to hemodynamic function and exercise economy.

The present study confirmed that our hypoxic training method effectively improved hemodynamic function and exercise economy by decreasing $\mathrm{HR}$ and $\mathrm{VO}_{2}$ and increasing SV during submaximal cycle ergometer exercise for 30 min versus normoxic training in amateur male swimmers. Hemodynamic function, including exercise economy and $\mathrm{VO}_{2 \max }$, are important factors that determine performance in endurance athletes [35-37]. Enhancing the exercise economy means improved adenosine triphosphate (ATP) resynthesis (per $1 \mathrm{~mol}$ of $\mathrm{O}_{2}$ ) and reduced ATP levels at a given exercise load [1,10,35]. In addition, the enhancement of exercise economy not only increases the efficiency of oxygen delivery and use capacity and energy availability; it also improves the invigoration of the PNS via activation of $\beta$-adrenergic receptors in the cardiac muscles and increases venous return, thereby effectively altering cardiac function $[1,10,12]$. Our study reported that aerobic continuous and interval training under hypoxia enhanced endurance exercise performance (e.g., $\mathrm{VO}_{2 \max }$ and 400-m time trial record) with improved exercise economy (decreased $\mathrm{VO}_{2}$ during submaximal exercise), venous return (decreased HR during submaximal exercise), and pumping ability of the heart (increased SV during submaximal exercise) than in normoxia in amateur male swimmers. These results are important for examining the effect of improving exercise performance through hypoxic training. 
ANS function can be quantitatively evaluated using HRV and reflects the interaction between the SNS and the PNS on the sinoatrial nodes, which regulate cardiovascular function [21]. HRV is the most effective and reproducible parameter among those obtained from tests for measuring changes in ANS function [38,39]. The HRV test has been mainly used in the fields of mental health examination and health science because it is highly correlated with mental disorders such as depression, anxiety, chronic fatigue syndrome, and insomnia [21,40]. In recent years, the HRV test has been widely used in the field of sports science to more effectively monitor the effectiveness and conditioning of exercise training $[10,23,39]$. Therefore, we confirmed the effect of aerobic continuous and interval training under hypoxia on ANS function using HRV tests versus normoxia in amateur male swimmers. The HTG showed a significantly improved RMSSD, TP, and LF/HF ratio, but no change was observed in the NTG.

RMSSD is the square root of the mean squared of successive NN interval differences, and it reflects short-term variability in heart rate and is mainly used to predict HRV in HF bands. RMSSD is an index that reflects the PNS and is interpreted as a physiologically healthy and relaxed state as its value increases [24]. TP is an indicator of the overall regulatory ability of the ANS. In the case of chronic stress or physical disease, TP decreases due to a reduction in the ability to regulate the ANS [41]. HF generally reflects the activity of the vagus nerve branching into the heart, while LF refers to the activity of the sympathetic nerve [40]. Therefore, the LF/HF ratio reflects the overall balance of the ANS, and a higher LF/HF ratio indicates that the SNS is relatively elevated or that the PNS activity is suppressed $[10,21]$. The present study confirmed that aerobic continuous and interval training under hypoxia can improve ANS function via parasympathetic activation and ANS balance by improving the RMSSD, TP, and LF/HF ratio. These results show that our hypoxic training program can improve HRV, that is, ANS function, to obtain a more efficient training effect, and it indicates that the exercise performance ability can be improved.

Among various hypoxic training methods, LLTH has received the most attention from various athletes because it commonly involves shorter exposure time to hypoxia (approximately three to five sessions per week of 1-3 h), less effort, lower cost, and less time $[1,10]$. In particular, CHT and IHT are most commonly used to enhance endurance exercise performance $[1,4,5,10]$. Many previous studies examined the exercise performance of swimmers via this type of hypoxic training. Park et al. [1] reported that high-intensity training ( $<3 \mathrm{~h}$ of hypoxic exposure 3 times per week for 6 weeks) composed of aerobic continuous treadmill and anaerobic interval bicycle exercise and normoxic training (swimming and resistance exercise) are effective at enhancing $\mathrm{VO}_{2 \max }$ and 400-m swimming performance via improved exercise economy in competitive swimmers. Truijens [17] tested the hypothesis that high-intensity hypoxic training improves sea-level performance more than equivalent training in normoxia. As a result, they concluded that five weeks of highintensity training in a flume improves sea-level swimming performance and $\mathrm{VO}_{2 \max }$ in well-trained swimmers with no additive effect of hypoxic training. Czuba [42] evaluated the efficacy of IHT on anaerobic and aerobic capacity and swimming performance in welltrained swimmers. They reported that high-intensity IHT is an effective training method for improving anaerobic capacity and swimming sprint performance. Park and Lim [4] evaluated the effects of six weeks of hypoxic training composed of warm-up, continuous training, interval training, elastic resistance training, and cool-down on exercise performance in moderately trained competitive swimmers. They proposed that hypoxic training is effective for improving muscular strength and endurance but resulted in unclear changes in $\mathrm{VO}_{2 \text { max }}$, anaerobic power, and 50-m and 400-m swimming performance compared to normoxic training.

Our hypoxic training method composed of aerobic continuous and interval training under hypoxia resulted in a clear enhancement of $\mathrm{VO}_{2 \max }$ and 400-m swimming record with improved hemodynamic function (decreased $\mathrm{HR}$ and $\mathrm{VO}_{2}$, increased SV) during submaximal exercise and ANS function (increased RMSSD and TP, decreased LF/HF ratio) in amateur male swimmers. Although the subjects of the present study were amateur male 
swimmers at a different initial level from previous studies of elite swimmers, our hypoxic training appears effective at enhancing endurance exercise performance, improvements that may be associated with improved hemodynamic and ANS function.

\section{Conclusions}

Our study demonstrated that six weeks of aerobic continuous and interval training under hypoxia enhanced endurance exercise performance versus normoxia in amateur male swimmers. In addition, the improvement in exercise performance (e.g., $\mathrm{VO}_{2 \max }$ and 400-m time trial record) observed after six weeks of hypoxic training might be due to the increase in hemodynamic function (e.g., $\mathrm{HR}, \mathrm{VO}_{2}$, and $\mathrm{SVi}$ ) during submaximal exercise and ANS function assessed by HRV.

Author Contributions: Study conception and design, H.-Y.P.; data curation, H.-Y.P.; formal analysis, S.-W.K., S.-S.N. and H.-Y.P.; investigation, S.-W.K., J.-W.K. and H.-Y.P.; methodology, S.-W.K., W.-S.J. and H.-Y.P.; writing — original draft, S.-W.K.; writing—review and editing, W.-S.J., J.-W.K., S.-S.N. and H.-Y.P. All authors have read and agreed to the published version of the manuscript.

Funding: This work was supported by the Ministry of Education of the Republic of Korea and the National Research Foundation of Korea (NRF-2019S1A5A2A03034583).

Institutional Review Board Statement: This study was approved by the Institutional Review Board of Konkuk University (7001355-201510-HR-090) in Korea and was conducted in accordance with the Declaration of Helsinki.

Informed Consent Statement: Informed consent was obtained from all subjects involved in the study.

Data Availability Statement: The data presented in this study are available on request from the corresponding author.

Conflicts of Interest: The authors declare no conflict of interest.

\section{References}

1. Park, H.Y.; Shin, C.; Lim, K. Intermittent hypoxic training for 6 weeks in $3000 \mathrm{~m}$ hypobaric hypoxia conditions enhances exercise economy and aerobic exercise performance in moderately trained swimmers. Biol. Sport 2018, 35, 49-56. [CrossRef]

2. Sinex, J.A.; Chapman, R.F. Hypoxic training methods for improving endurance exercise performance. J. Sport Health Sci. 2015, 4, 325-332. [CrossRef]

3. Millet, G.P.; Faiss, R.; Brocherie, F.; Girard, O. Hypoxic training and team sports: A challenge to traditional methods? Br. J. Sports Med. 2013, 47 (Suppl. 1), i6-i7. [CrossRef]

4. Park, H.Y.; Lim, K. Effects of hypoxic training versus normoxic training on exercise performance in competitive swimmers. J. Sports Sci. Med. 2017, 16, 480-488.

5. Czuba, M.; Waskiewicz, Z.; Zajac, A.; Poprzecki, S.; Cholewa, J.; Roczniok, R. The effects of intermittent hypoxic training on aerobic capacity and endurance performance in cyclists. J. Sports Sci. Med. 2011, 10, 175-183.

6. Wilber, R.L. Application of altitude/hypoxic training by elite athletes. Med. Sci. Sports Exerc. 2007, 39, 1610-1624. [CrossRef]

7. Park, H.Y.; Hwang, H.; Park, J.; Lee, S.; Lim, K. The effects of altitude/hypoxic training on oxygen delivery capacity of the blood and aerobic exercise capacity in elite athletes-A meta-analysis. J. Exerc. Nutr. Biochem. 2016, 20, 15-22. [CrossRef]

8. Levine, B.D.; Stray-Gundersen, J. "Living high-training low": Effect of moderate-altitude acclimatization with low-altitude training on performance. J. Appl. Physiol. 1997, 83, 102-112. [CrossRef]

9. Park, H.Y.; Park, W.; Lim, K. Living high-training low for 21 days enhances exercise economy, hemodynamic function, and exercise performance of competitive runners. J. Sports Sci. Med. 2019, 18, 427-437.

10. Jung, W.S.; Kim, S.W.; Park, H.Y. Interval hypoxic training enhances athletic performance and does not adversely affect immune function in middle- and long-distance runners. Int. J. Environ. Res. Public Health 2020, 17, 1934. [CrossRef]

11. Girard, O.; Brocherie, F.; Goods, P.S.R.; Millet, G.P. An updated panorama of "living low-training high" altitude/hypoxic methods. Front. Sports Act. Living 2020, 2, 26. [CrossRef] [PubMed]

12. Park, H.Y.; Jung, W.S.; Kim, J.; Hwang, H.; Lim, K. Efficacy of intermittent hypoxic training on hemodynamic function and exercise performance in competitive swimmers. J. Exerc. Nutr. Biochem. 2018, 22, 32-38. [CrossRef] [PubMed]

13. Hamlin, M.J.; Marshall, H.C.; Hellemans, J.; Ainslie, P.N.; Anglem, N. Effect of intermittent hypoxic training on $20 \mathrm{~km}$ time trial and $30 \mathrm{~s}$ anaerobic performance. Scand. J. Med. Sci. Sports 2010, 20, 651-661. [CrossRef] [PubMed]

14. Rodríguez, F.A.; Truijens, M.J.; Townsend, N.E.; Stray-Gundersen, J.; Gore, C.J.; Levine, B.D. Performance of runners and swimmers after four weeks of intermittent hypobaric hypoxic exposure plus sea level training. J. Appl. Physiol. 2007, 103, 1523-1535. [CrossRef] [PubMed] 
15. Roels, B.; Bentley, D.J.; Coste, O.; Mercier, J.; Millet, G.P. Effects of intermittent hypoxic training on cycling performance in well-trained athletes. Eur. J. Appl. Physiol. 2007, 101, 359-368. [CrossRef]

16. Hendriksen, I.J.; Meeuwsen, T. The effect of intermittent training in hypobaric hypoxia on sea-level exercise: A cross-over study in humans. Eur. J. Appl. Physiol. 2003, 88, 396-403. [CrossRef]

17. Truijens, M.J.; Toussaint, H.M.; Dow, J.; Levine, B.D. Effect of high-intensity hypoxic training on sea-level swimming performances. J. Appl. Physiol. 2003, 94, 733-743. [CrossRef]

18. Krassioukov, A.; West, C. The role of autonomic function on sport performance in athletes with spinal cord injury. Pmr 2014, 6, S58-S65. [CrossRef]

19. Vitale, J.A.; Bonato, M.; La Torre, A.; Banfi, G. Heart rate variability in sport performance: Do time of day and chronotype play a role? J. Clin. Med. 2019, 8, 723. [CrossRef]

20. Hautala, A.J.; Kiviniemi, A.M.; Tulppo, M.P. Individual responses to aerobic exercise: The role of the autonomic nervous system. Neurosci. Biobehav. Rev. 2009, 33, 107-115. [CrossRef]

21. Park, H.Y.; Jung, W.S.; Kim, J.; Lim, K. Twelve weeks of exercise modality in hypoxia enhances health-related function in obese older Korean men: A randomized controlled trial. Geriatr. Gerontol. Int. 2019, 19, 311-316. [CrossRef]

22. Park, H.Y.; Jung, W.S.; Kim, J.; Hwang, H.; Lim, K. Twelve weeks of aerobic exercise at the lactate threshold improves autonomic nervous system function, body composition, and aerobic performance in women with obesity. J. Obes. Metab. Syndr. 2020, 29, 67-75. [CrossRef]

23. Herzig, D.; Asatryan, B.; Brugger, N.; Eser, P.; Wilhelm, M. The association between endurance training and heart rate variability: The confounding role of heart rate. Front. Physiol. 2018, 9, 756. [CrossRef]

24. Dong, J.G. The role of heart rate variability in sports physiology. Exp. Ther. Med. 2016, 11, 1531-1536. [CrossRef]

25. Gore, C.J.; Rodríguez, F.A.; Truijens, M.J.; Townsend, N.E.; Stray-Gundersen, J.; Levine, B.D. Increased serum erythropoietin but not red cell production after $4 \mathrm{wk}$ of intermittent hypobaric hypoxia (4000-5500 m). J. Appl. Physiol. 2006, 101, 1386-1393. [CrossRef]

26. Gore, C.J.; Sharpe, K.; Garvican-Lewis, L.A.; Saunders, P.U.; Humberstone, C.E.; Robertson, E.Y.; Wachsmuth, N.B.; Clark, S.A.; McLean, B.D.; Friedmann-Bette, B.; et al. Altitude training and haemoglobin mass from the optimised carbon monoxide rebreathing method determined by a meta-analysis. Br. J. Sports Med. 2013, 47 (Suppl. S1), i31-i39. [CrossRef]

27. Bonetti, D.L.; Hopkins, W.G. Sea-level exercise performance following adaptation to hypoxia: A meta-analysis. Sports Med. 2009, 39, 107-127. [CrossRef]

28. Ge, R.L.; Witkowski, S.; Zhang, Y.; Alfrey, C.; Sivieri, M.; Karlsen, T.; Resaland, G.K.; Harber, M.; Stray-Gundersen, J.; Levine, B.D. Determinants of erythropoietin release in response to short-term hypobaric hypoxia. J. Appl. Physiol. 2002, 92, $2361-2367$. [CrossRef]

29. Park, H.-Y.; Kim, J.; Lim, K. Exercise physiology basis and necessity of hypoxic training to improve exercise performance in elite athletes. Korean J. Sport Sci. 2018, 29, 737-752.

30. Płoszczyca, K.; Langfort, J.; Czuba, M. The effects of altitude training on erythropoietic response and hematological variables in adult athletes: A narrative review. Front. Physiol. 2018, 9, 375. [CrossRef]

31. Park, H.Y.; Jung, W.S.; Kim, J.; Hwang, H.; Kim, S.W.; An, Y.; Lee, H.; Jeon, S.; Lim, K. Effects of 2-week exercise training in hypobaric hypoxic conditions on exercise performance and immune function in Korean national cycling athletes with disabilities: A case report. Int. J. Environ. Res. Public Health 2020, 17, 861. [CrossRef] [PubMed]

32. Faiss, R.; Girard, O.; Millet, G.P. Advancing hypoxic training in team sports: From intermittent hypoxic training to repeated sprint training in hypoxia. Br. J. Sports Med. 2013, 47 (Suppl. S1), i45-i50. [CrossRef]

33. Levine, B.D.; Stray-Gundersen, J. Point: Positive effects of intermittent hypoxia (live high:train low) on exercise performance are mediated primarily by augmented red cell volume. J. Appl. Physiol. 2005, 99, 2053-2055. [CrossRef] [PubMed]

34. Millet, G.P.; Boissiere, D.; Candau, R. Energy cost of different skating techniques in cross-country skiing. J. Sports Sci 2003, $21,3-11$. [CrossRef] [PubMed]

35. Green, H.J.; Roy, B.; Grant, S.; Hughson, R.; Burnett, M.; Otto, C.; Pipe, A.; McKenzie, D.; Johnson, M. Increases in submaximal cycling efficiency mediated by altitude acclimatization. J. Appl. Physiol. 2000, 89, 1189-1197. [CrossRef]

36. Saunders, P.U.; Telford, R.D.; Pyne, D.B.; Cunningham, R.B.; Gore, C.J.; Hahn, A.G.; Hawley, J.A. Improved running economy in elite runners after 20 days of simulated moderate-altitude exposure. J. Appl. Physiol. 2004, 96, 931-937. [CrossRef]

37. Gore, C.J.; Hahn, A.G.; Aughey, R.J.; Martin, D.T.; Ashenden, M.J.; Clark, S.A.; Garnham, A.P.; Roberts, A.D.; Slater, G.J.; McKenna, M.J. Live high:train low increases muscle buffer capacity and submaximal cycling efficiency. Acta Physiol. Scand. 2001, 173, 275-286. [CrossRef]

38. Alansare, A.; Alford, K.; Lee, S.; Church, T.; Jung, H.C. The effects of high-intensity interval training vs. moderate-intensity continuous training on heart rate variability in physically inactive adults. Int. J. Environ. Res. Public Health 2018, $15,1508$. [CrossRef]

39. Plews, D.J.; Laursen, P.B.; Stanley, J.; Kilding, A.E.; Buchheit, M. Training adaptation and heart rate variability in elite endurance athletes: Opening the door to effective monitoring. Sports Med. 2013, 43, 773-781. [CrossRef]

40. Kim, J.; Park, H.Y.; Lim, K. Effects of 12 weeks of combined exercise on heart rate variability and dynamic pulmonary function in obese and elderly Korean women. Iran. J. Public Health 2018, 47, 74-81. 
41. Assoumou, H.G.; Pichot, V.; Barthelemy, J.C.; Dauphinot, V.; Celle, S.; Gosse, P.; Kossovsky, M.; Gaspoz, J.M.; Roche, F. Metabolic syndrome and short-term and long-term heart rate variability in elderly free of clinical cardiovascular disease: The PROOF study. Rejuvenation Res. 2010, 13, 653-663. [CrossRef] [PubMed]

42. Czuba, M.; Wilk, R.; Karpiński, J.; Chalimoniuk, M.; Zajac, A.; Langfort, J. Intermittent hypoxic training improves anaerobic performance in competitive swimmers when implemented into a direct competition mesocycle. PLoS ONE 2017, 12, e0180380. [CrossRef] [PubMed] 